\title{
Regional variation in letter joining habits in Arabic writers
}

\begin{abstract}
This paper describes the ways in which a number of letter pairs written in Arabic can be constructed, classifying them in different ways according to the fine detail of the pen movements involved. Variations of writing habit between writers from four countries (Oman, Jordan, Tunisia and Morocco) are shown. Such studies generally focus on individual letterforms, but this study is original in its focusing on the method of joining letters. National differences were found and these could provide useful information when examining unknown handwritings of people from these places for example by forensic handwriting experts.
\end{abstract}

Keywords: Greek, Arabic, Chinese, Omani writers
Volume 4 Issue 2 - 2017

\author{
Ahmed AN Al Hadhrami \\ Royal Oman Police, Oman
}

Correspondence: Ahmed AN Al Hadhrami, Royal Oman Police, PO Box I3I, PC. I I 5, Madinat al Sultan Qaboos, Muscat, Oman, Tel 96899436536, Fax 96824562993 ,

Email gaith1994@yahoo.com, ahmed.alhadhrami@yahoo.com

Received: January 23, 2017| Published: February 20, 2017

\section{Introduction}

Handwriting is a complex skill requiring high level cognitive and motor control. ${ }^{1}$ The difficult integration of both the cognitive and motor components, even in skilful writers, is such that handwriting is constantly varying from one occasion to another producing what is usually called natural variation. The variations in handwriting are an important aspect of a particular writer's handwriting above and beyond the various letterforms that writer uses. In short, variability is an intrinsic and inescapable dimension to handwriting production. An understanding of handwriting variability is central to the work of handwriting experts whose task it is to help to identify handwriting of unknown origin. Handwriting is generally taught to children typically in a school environment when they are around the age of five or six. The teaching period lasts several years and even when formal teaching stops, the individual's handwriting continues to develop into early adulthood. ${ }^{2}$ Handwriting in a particular system, be it using the Roman alphabet used in most European languages, Greek, Arabic, Chinese or some other system, requires a degree of conforming to expected letterforms so as to be readable by others. However, the styles that are taught vary from place to place and over time ${ }^{3}$ which leads to the possibility of being able to perhaps identifying where and when a writer learned to write based on the particular features included in their handwriting. This assumes that the styles taught to the young child, despite personal modification by the individual as their handwriting develops, will still be present (albeit possibly modified) and recognisable in their adult handwriting.

Studies of regional and national habits in handwriting production have demonstrated that letterforms will often vary according to where the writer learns their handwriting skills. For example Turnbull et al for Polish writers ${ }^{4}$ and Cheng et al., ${ }^{5}$ for Chinese, Malay and Indian writers in Singapore $\left.{ }^{5}\right]$ showed such an effect the causes of which are more difficult to isolate but which must reflect educational and cultural practices in different countries and regions. Al-Hadhrami and colleagues $^{6}$ showed a similar trend for Arabic writers from Morocco, Tunisia, Jordan and Oman based on several letterforms studied. Further, the effects were even more marked when the two North African countries (Morocco and Tunisia) were compared to the two Middle Eastern countries (Oman and Jordan) suggesting that regional effects of handwriting variation might be even stronger than national ones. The focus in all such studies has been on letterforms rather than other aspects of handwriting formation. This study will consider the ways in which certain Arabic letter pairs are joined to one another. The letters in this study of joining habits were all classified as connected letters in the earlier paper. ${ }^{6}$ This paper will describe the different ways in which a number of letter pair combinations can be constructed and how these possible joining methods vary from country to country.

\section{Method}

Fifty samples of handwriting from each of Morocco, Tunisia, Jordan and Oman were examined. The samples in this study were selected at random from the larger sample pool (150 samples of handwriting) used in the study of letterforms in Arabic writers from these countries. ${ }^{6}$ Arabic is written from right to left and each of the letter. Each of the letter six pairs listed below can be joined in one of three ways, namely at the top, bottom, or front of the letters or, a fourth category, is where the letters are unjoined. The particular letter pairs were chosen as these characters are complex in their formation which increases the possibility of variations in the way the characters are joined. In general, the more complex the characters are in their formation, the more variation will occur between people in the way of connecting them. Further, the letter pairs occur frequently in Arabic writing and were present in the texts available from the previous study. ${ }^{6}$ This is important because letter combinations that are not frequently encountered may be more likely to vary both within the handwriting of a particular person and between different writers as its very unfamiliarity may cause it to be a less automatic, natural handwriting movement. ${ }^{7}$ The six letter pairs are shown in Figure 1. 


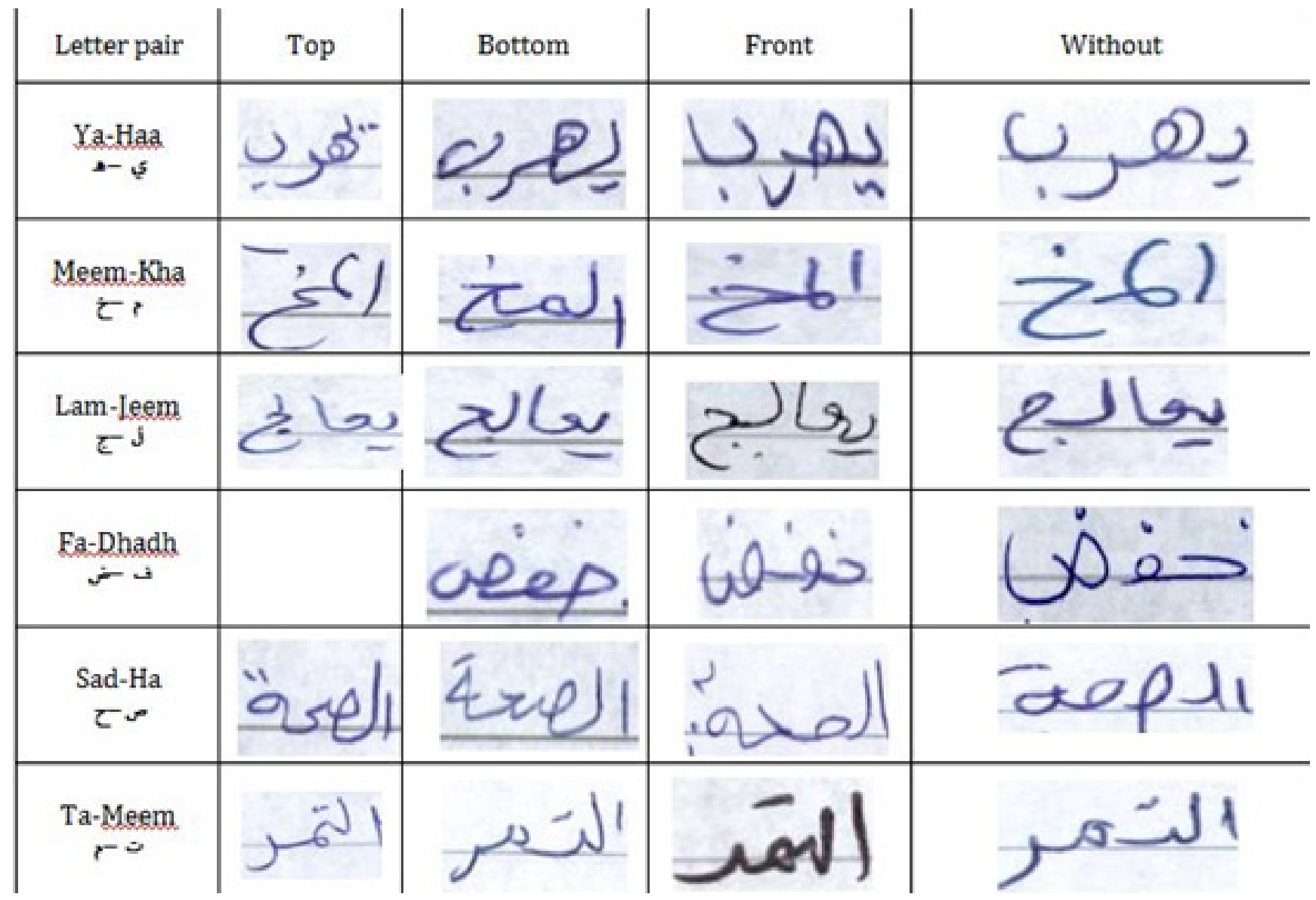

Figure I Showing examples of the four types of joining habit for the six letter pairs studied.

\section{Results and discussion}

Each letter pair was examined with a view to determining the method of construction of the join. The letterforms studied were such that the joining could be considered to be either at the top of the character, the bottom of the character, the front of the character or without joining. The frequency of the constructions for the letter pairs are shown country by country in Tables $1-4$. The data in Tables 1-4 show two main trends. Firstly, that the method of joining is carried across between letters pairs in a given location. For example, in Tunisia, the letter pairs studied are frequently in the without category irrespective of the particular letter pairing. Secondly, the habits vary from country to country, for example the without category that is so often found in Tunisian writers is much more rarely found among Omani writers. The main value of the data in Tables $1-4$ as that enables a handwriting expert to assess the likely place where a writer learned to write based on the combination of features found in a piece of handwriting. Such assessments must always be treated with caution but it may assist an investigator to know the place from which a writer originates and also may assist the handwriting expert to take account of the features found as to whether they are more or less frequently found in particular populations of writers. In this way, a 'catalogue' of handwriting features can be built up in various writing systems in the countries of the world to show how the various styles of handwriting vary across the globe.
Table I Showing joining frequencies of writers from Oman

\begin{tabular}{lllll}
\hline Letter Pair & Top & Bottom & Front & Without \\
\hline Ya-Haa & 6 & 20 & 22 & 2 \\
Meem-Kha & 7 & 19 & 7 & 17 \\
Lam-Jim & 18 & 15 & 7 & 10 \\
Fa-Dhadh & 0 & 27 & 9 & 14 \\
Sad-Ha & 17 & 15 & 8 & 10 \\
Ta-Meem & 32 & 1 & 16 & 1 \\
\hline
\end{tabular}

Table 2 Showing joining frequencies of writers from Jordan

\begin{tabular}{lllll}
\hline Letter Pair & Top & Bottom & Front & Without \\
\hline Ya-Haa & 10 & 13 & 27 & 0 \\
Meem-Kha & 15 & 19 & 8 & 8 \\
Lam-Jim & 27 & 15 & 7 & 1 \\
Fa-Dhadh & 0 & 41 & 7 & 2 \\
Sad-Ha & 37 & 8 & 2 & 3 \\
Ta-Meem & 40 & 3 & 7 & 0 \\
\hline
\end{tabular}


Table 3 Showing joining frequencies of writers from Morocco

\begin{tabular}{lllll}
\hline Letter Pair & Top & Bottom & Front & Without \\
\hline Ya-Haa & 8 & 33 & 9 & 0 \\
Meem-Kha & 2 & 17 & 6 & 25 \\
Lam-Jim & 27 & 15 & 7 & 1 \\
Fa-Dhadh & 0 & 14 & 18 & 18 \\
Sad-Ha & 2 & 16 & 4 & 28 \\
Ta-Meem & 4 & 13 & 24 & 9 \\
\hline
\end{tabular}

Table 4 Showing joining frequencies of writers from Tunisia

\begin{tabular}{lllll}
\hline Letter Pair & Top & Bottom & Front & Without \\
\hline Ya-Haa & 0 & 6 & 40 & 4 \\
Meem-Kha & 6 & 21 & 3 & 20 \\
Lam-Jim & 12 & 26 & 3 & 9 \\
Fa-Dhadh & 0 & 26 & 8 & 16 \\
Sad-Ha & 6 & 28 & 1 & 15 \\
Ta-Meem & 4 & 15 & 23 & 8 \\
\hline
\end{tabular}

\section{Conclusion}

The forensic examination of handwriting is a skill that requires that the expert has relevant knowledge of the variability of handwriting styles and features for the place where they work and, in an increasingly cosmopolitan world, at least an awareness that writers taught in different places may show different combinations of handwriting features. This study adds more into the growing pool of knowledge showing how different combinations of handwriting features can provide important information to those studying handwriting of unknown origin.

\section{Acknowledgments}

None.

\section{Conflicts of interest}

None.

\section{References}

1. Van Galen GP. Handwriting: Issues for a psychomotor theory. Human Movement Science. 1991;10(2-3):165-191.

2. Allen M. Developmental aspects of handwriting acquisition. 2011;1-352.

3. Huber RA, Headrick AM. Handwriting identification: Facts and fundamentals. 1st edn. 1999;1-434.

4. Turnbull SJ, Jones AE, Allen M. Identification of the Class Characteristics in the Handwriting of Polish People Writing in English. J Forensic Sci. 2010;55(5):1296-1303.

5. Cheng N, Lee GK, Yap BS, et al. Investigation of class characteristics in English handwriting of the three main racial groups: Chinese, Malay and Indian in Singapore. J Forensic Sci. 2005;50(1):177-184.

6. Al Hadhrami AA, Allen M, Moffatt C, et al. National characteristics and variation in Arabic handwriting. Forensic Sci Int. 2015;247:89-96.

7. Graham S, Struck M, Santoro J, et al. Dimensions of good, and poor handwriting legibility in first and second graders: Motor programs, visual-spatial arrangement, and letter formation parameter setting. Dev Neuropsychol. 2006;29(1):43-60. 